\title{
Correction to: Coaseian Biodiversity Conservation and Market Power
}

\author{
Thomas Eichner $^{1}$ • Rüdiger Pethig ${ }^{2}$
}

Published online: 5 March 2018

(C) Springer Science+Business Media B.V., part of Springer Nature 2018

\section{Correction to: Environ Resource Econ https://doi.org/10.1007/s10640-018-0225-0}

This erratum corrects an error in that part of the article, which relates to the exertion of market power. Equations (C3), (C7) and (C8) in "Appendix" should be replaced by

$$
\begin{aligned}
& p_{x}=P^{x}\left[\left(b_{i}\right)_{i \in \mathcal{N}}\right]=a_{x}-\frac{2 \alpha_{x} \beta_{x}}{n+s} \sum_{\mathcal{N}} \sqrt{\ell-b_{i}}, \\
& p_{y}=P^{y}\left[\left(b_{j}\right)_{j \in \mathcal{S}}\right]=a_{y}-\frac{2 \alpha_{y} \beta_{y}}{n+s} \sum_{\mathcal{S}} \sqrt{\ell-b_{j}}, \\
& p_{x}=P^{x}\left[\left(z_{i}\right)_{i \in \mathcal{N}}\right]=a_{x}-\frac{2 \alpha_{x} \beta_{x}}{n+s} \sum_{\mathcal{N}} \sqrt{\ell-{ }_{1} b_{i}-z_{i}}, \\
& p_{y}=P^{y}\left[\left(z_{j}\right)_{j \in \mathcal{S}}\right]=a_{y}-\frac{2 \alpha_{y} \beta_{y}}{n+s} \sum_{\mathcal{S}} \sqrt{\ell-{ }_{1} b_{j}-z_{j}},
\end{aligned}
$$

and in the first-order conditions (C5), (C6), (C10), (C11), (C13) and (C14) the differential quotients $\frac{\partial P^{x}}{\partial b_{\mathcal{N}}}, \frac{\partial P^{y}}{\partial b_{\mathcal{S}}}, \frac{\partial P^{x}}{\partial z_{\mathcal{N}}}, \frac{\partial P^{y}}{\partial z_{\mathcal{S}}}$ should be replaced by $\frac{\partial P^{x}}{\partial b_{i}}, \frac{\partial P^{y}}{\partial b_{j}}, \frac{\partial P^{x}}{\partial z_{i}}, \frac{\partial P^{y}}{\partial z_{j}}$. While the analysis and the numerical simulations in the regimes without strategy remain unchanged, the correct numbers in the regimes with strategies are listed in Tables 1, 2 and 3 below. These tables replace the last three rows of the Tables 8, 9 and 10, respectively, in "Appendix" of the article.

The original article can be found online at https://doi.org/10.1007/s10640-018-0225-0.

$\triangle$ Thomas Eichner

thomas.eichner@fernuni-hagen.de

Rüdiger Pethig

pethig@vwl.wiwi.uni-siegen.de

1 Department of Economics, University of Hagen, Universitätsstr. 41, 58097 Hagen, Germany

2 Department of Economics, University of Siegen, Unteres Schloss 3, 57072 Siegen, Germany 
Table 1 Equilibrium values in Example $1\left(\beta_{x}=0.1\right)$

\begin{tabular}{lccccccccc}
\hline & $\sum_{\Omega}{ }_{k} b_{h}$ & ${ }_{k} b_{\mathcal{N}}$ & ${ }_{k} b_{\mathcal{S}}$ & $\sum_{\Omega}{ }_{k} w_{h}$ & ${ }_{k} w_{\mathcal{N}}$ & ${ }_{k} w_{\mathcal{S}}$ & ${ }_{k} p_{x}$ & ${ }_{k} p_{y}$ & ${ }_{k} g^{d}-{ }_{k} G_{\mathcal{N}}$ \\
\hline${\text { Regime }{ }^{s}}^{1536.17}$ & 10.11 & 20.61 & $1,859,470$ & $19,204.8$ & $17,984.6$ & 949.05 & 891.09 & 1058.29 \\
Regime 2 $^{s}$ & 1544.02 & 10.11 & 20.77 & $1,859,510$ & $19,205.5$ & $17,984.7$ & 949.05 & 891.10 & 1066.05 \\
Regime 3 $^{s}$ & 2314.89 & 18.37 & 27.92 & $1,861,430$ & $19,222.1$ & $18,006.5$ & 949.10 & 891.51 & 969.54 \\
\hline
\end{tabular}

Table 2 Equilibrium values in Example $2\left(\alpha_{y}=0.9\right)$

\begin{tabular}{llllllllll}
\hline & $\sum_{\Omega}{ }_{k} b_{h}$ & ${ }_{k} b_{\mathcal{N}}$ & ${ }_{k} b_{\mathcal{S}}$ & $\sum_{\Omega}{ }_{k} w_{h}$ & ${ }_{k} w_{\mathcal{N}}$ & ${ }_{k} w_{\mathcal{S}}$ & ${ }_{k} p_{x}$ & ${ }_{k} p_{y}$ & ${ }_{k} g^{d}-{ }_{k} G_{\mathcal{N}}$ \\
\hline Regime 1 $^{s}$ & 2278.11 & 10.11 & 35.45 & $1,787,600$ & $19,265.4$ & $16,486.6$ & 949.05 & 892.77 & 2542.43 \\
Regime 2 $^{s}$ & 2284.50 & 10.11 & 35.58 & $1,787,635$ & $19,266.0$ & $16,486.7$ & 949.05 & 892.78 & 2548.76 \\
Regime 3 $^{s}$ & 2989.02 & 18.37 & 41.41 & $1,789,390$ & $19,284.0$ & $16,503.8$ & 949.10 & 893.11 & 2392.23 \\
\hline
\end{tabular}

Table 3 Equilibrium values in Example $3\left(\beta_{x}=0.5\right)$

\begin{tabular}{llllllllll}
\hline & $\sum_{\Omega}{ }_{k} b_{h}$ & ${ }_{k} b_{\mathcal{N}}$ & ${ }_{k} b_{\mathcal{S}}$ & $\sum_{\Omega}{ }_{k} w_{h}$ & ${ }_{k} w_{\mathcal{N}}$ & ${ }_{k} w_{\mathcal{S}}$ & ${ }_{k} p_{x}$ & ${ }_{k} p_{y}$ & ${ }_{k} g^{d}-{ }_{k} G_{\mathcal{N}}$ \\
\hline Regime 1 $^{s}$ & 1572.20 & 10.83 & 20.61 & $1,857,855$ & $19,154.7$ & $18,002.4$ & 945.28 & 891.09 & 986.52 \\
Regime 2 $^{s}$ & 1580.04 & 10.83 & 20.77 & $1,857,895$ & $19,155.4$ & $18,002.5$ & 945.28 & 891.10 & 994.28 \\
Regime 3 $^{s}$ & 2361.07 & 19.30 & 27.92 & $1,859,800$ & $19,173.4$ & $18,022.6$ & 945.51 & 891.51 & 888.66 \\
\hline
\end{tabular}

The correct strategic effects turn out to be smaller than shown in Tables 8, 9, and 10 of the article. However, apart from minor deviations the economic interpretation of Examples 1 and 2 with strategy is the same as in the article. When the correct numbers of Table 3 below are applied, the interpretation of Example 3 with strategy is qualitatively the same as that of Examples 1 and 2. 